\title{
The Interval-Valued Intuitionistic Fuzzy Optimized Weighted Bonferroni Means and Their Application
}

\author{
Ya-ming Shi and Jian-min He \\ School of Economics and Management, Southeast University, Nanjing 211189, China \\ Correspondence should be addressed to Ya-ming Shi; 55658741@qq.com
}

Received 3 May 2013; Accepted 18 June 2013

Academic Editor: Guangchen Wang

Copyright (C) 2013 Y.-m. Shi and J.-m. He. This is an open access article distributed under the Creative Commons Attribution License, which permits unrestricted use, distribution, and reproduction in any medium, provided the original work is properly cited.

\begin{abstract}
We investigate and propose two new Bonferroni means, that is, the optimized weighted BM (OWBM) and the generalized optimized weighted BM (GOWBM), whose characteristics are to reflect the preference and interrelationship of the aggregated arguments and can satisfy the basic properties of the aggregation techniques simultaneously. Further, we propose the intervalvalued intuitionistic fuzzy optimized weighted Bonferroni mean (IIFOWBM) and the generalized interval-valued intuitionistic fuzzy optimized weighted Bonferroni mean (GIIFOWBM) and detailed study of their desirable properties such as idempotency, monotonicity, transformation, and boundary. Finally, based on IIFOWBM and GIIFOWBM, we give an approach to group decision making under the interval-valued intuitionistic fuzzy environment and utilize a practical case involving the assessment of a set of agroecological regions in Hubei Province, China, to illustrate the developed methods.
\end{abstract}

\section{Introduction}

As a useful aggregation technique, the Bonferroni mean (BM) can capture the interrelationship between input arguments and has been a hot research topic recently. Bonferroni [1] originally introduced a mean-type aggregation operator, called the Bonferroni mean, whose prominent characteristic is that it cannot only consider the importance of each criterion but also reflect the interrelationship of the individual criterion. Recently, Yager [2] extended the BM by two mean-type operators, such as the Choquet integral operator [3] and the ordered weighted averaging operator [4], as well as associates differing importance with the arguments. Mordelová and Rückschlossová [5] also investigated the generalizations of $\mathrm{BM}$ referred to as $\mathrm{ABC}$-aggregation functions. Beliakov et al. [6] further extended the BM by considering the correlations of any three aggregated arguments instead of any two and proposed the generalized Bonferroni mean (GBM).

Nevertheless, the arguments suitable to be aggregated by the BM and GBM can only take the forms of crisp numbers. In the real world, due to the increasing complexity of the socioeconomic environment and the lack of knowledge and data, crisp data are sometimes unavailable. Thus, the input arguments may be more suitable with representation of fuzzy formats, such as fuzzy number [7], interval-valued fuzzy number [8], intuitionistic fuzzy value [9], interval-valued intuitionistic fuzzy value [10], and hesitant fuzzy element [11]. Thus, $\mathrm{Xu}$ and Yager [12] introduced the intuitionistic fuzzy Bonferroni mean (IFBM) and the intuitionistic fuzzy weighted Bonferroni mean (IFWBM). Xu and Chen [13] further proposed the interval-valued intuitionistic fuzzy Bonferroni mean (IIFBM) and the interval-valued intuitionistic fuzzy weighted Bonferroni mean (IIFWBM). Xia et al. [14] proposed the generalized intuitionistic fuzzy Bonferroni means. Zhou and He [15] developed some geometric Bonferroni Means. Furthermore, Beliakov and James [16] defined Bonferroni means over lattices which is a new viewpoint. Recently, Zhou and $\mathrm{He}$ [17] constructed an intuitionistic fuzzy weighted Bonferroni mean, Xia et al. [18] further investigated the generalized geometric Bonferroni means, Beliakov and James [19] extend the generalized Bonferroni means to Atanassov orthopairs, and so on.

The desirable characteristic of the BM is its capability to capture the interrelationship between input arguments. However, the classical BM and GBM, even the extended BMs, cannot reflect the interrelationship between the individual 
criterion and other criteria. To deal with these issues, in this paper, we propose the optimized weighted Bonferroni mean (OWBM) and the generalized optimized weighted Bonferroni mean (GOWBM), whose characteristics are to reflect the preference and interrelationship of the aggregated arguments and can satisfy the basic properties of the aggregation techniques simultaneously. Further, we have proposed the interval-valued intuitionistic fuzzy optimized weighted Bonferroni mean (IIFOWBM) and the generalized intervalvalued intuitionistic fuzzy optimized weighted Bonferroni mean (GIIFOWBM) and detailed study of their desirable properties such as idempotency, monotonicity, transformation, and boundary.

The remainder of this paper is organized as follows. We briefly review some basic definitions of the intervalvalued intuitionistic fuzzy values and Bonferroni mean. Then, in Section 3, we propose two BMs including OWBM and GOWBM and study their properties. Furthermore, in Section 4, we develop the IIFOWBM and GIIFOWBM operators, and their idempotency, monotonicity, transformation, and boundary are also investigated. A practical example is provided in Section 5 to demonstrate the application of the OWBM, GOWBM, and two interval-valued intuitionistic fuzzy BMs. The paper ends in Section 6 with concluding remarks.

\section{Some Basic Concepts}

2.1. Interval-Valued Intuitionistic Fuzzy Values. In the following, we introduce the basic concepts and operations related to interval-valued intuitionistic fuzzy value [20], which is an extended definition of the intuitionistic fuzzy value and interval-valued intuitionistic fuzzy set $[21,22]$.

Definition 1 (see [10]). Let $X=\left(x_{1}, x_{2}, \ldots, x_{n}\right)$ be fixed. An interval-valued intuitionistic fuzzy set (IIFS) $\widetilde{A}$ in $X$ can be defined as

$$
\widetilde{A}=\left\{\left(x, \widetilde{\mu}_{\widetilde{A}}(x), \widetilde{v}_{\widetilde{A}}(x)\right) \mid x \in X\right\},
$$

where $\widetilde{\mu}_{\widetilde{A}}(x) \subset[0,1]$ and $\widetilde{\nu}_{\widetilde{A}}(x) \subset[0,1]$ satisfy sup $\widetilde{\mu}_{\widetilde{A}}(x)+$ $\sup \widetilde{v}_{\widetilde{A}}(x) \leq 1$ for all $x_{i} \in X$ and $\widetilde{\mu}_{\widetilde{A}}(x)$ and $\widetilde{v}_{\widetilde{A}}(x)$ are, respectively, called the degree of membership and the degree of nonmembership of the element $x_{i} \in X$ to $A$.

Definition 2 (see [20]). Let $\widetilde{A}=\left\{\left(x, \widetilde{\mu}_{\widetilde{A}}(x), \widetilde{\nu}_{\widetilde{A}}(x)\right) \mid x \in X\right\}$ be an IIFS; the pair $\left(\widetilde{\mu}_{\widetilde{A}}(x), \widetilde{v}_{\widetilde{A}}(x)\right)$ is called an interval-valued intuitionistic fuzzy value (IIFV).

For computational convenience, an IIFV can be denoted by $([a, b],[c, d])$, with the condition that $[a, b] \subset[0,1]$, $[c, d] \subset[0,1]$, and $b+d \leq 1$. Furthermore, based on the operations of interval-valued intuitionistic fuzzy values $[21,22], \mathrm{Xu}$ [23] defined some operations of IIFNs as follows.

Definition 3 (see [23]). Letting $\tilde{\alpha}=([a, b],[c, d]), \widetilde{\alpha}_{1}=$ $\left(\left[a_{1}, b_{1}\right],\left[c_{1}, d_{1}\right]\right)$, and $\widetilde{\alpha}_{2}=\left(\left[a_{2}, b_{2}\right],\left[c_{2}, d_{2}\right]\right)$ be three IIFVs, then the following operational laws are valid:

(1) $\tilde{\alpha}_{1} \oplus \tilde{\alpha}_{2}=\left(\left[a_{1}+a_{2}-a_{1} a_{2}, b_{1}+b_{2}-b_{1} b_{2}\right],\left[c_{1} c_{2}, d_{1} d_{2}\right]\right)$,
(2) $\tilde{\alpha}_{1} \otimes \widetilde{\alpha}_{2}=\left(\left[a_{1} a_{2}, b_{1} b_{2}\right],\left[c_{1}+c_{2}-c_{1} c_{2}, d_{1}+d_{2}-d_{1} d_{2}\right]\right)$,

(3) $\lambda \widetilde{\alpha}=\left(\left[1-(1-a)^{\lambda}, 1-(1-b)^{\lambda}\right],\left[c^{\lambda}, d^{\lambda}\right]\right), \lambda>0$,

(4) $\widetilde{\alpha}^{\lambda}=\left(\left[a^{\lambda}, b^{\lambda}\right],\left[1-(1-c)^{\lambda}, 1-(1-d)^{\lambda}\right]\right), \lambda>0$.

Then, $\mathrm{Xu}$ [23] introduced the score function $s(\widetilde{\alpha})=(a-$ $c+b-d) / 2$ and the accuracy function $h(\widetilde{\alpha})=(a+b+c+d) / 2$ to calculate the score value and accuracy degree of IIFV $\widetilde{\alpha}=$ $([a, b],[c, d])$ and gave an order relation between two IIFNs $\widetilde{\alpha}_{1}$ and $\widetilde{\alpha}_{2}$ as follows:

(1) if $s\left(\widetilde{\alpha}_{1}\right)<s\left(\widetilde{\alpha}_{2}\right)$, then $\widetilde{\alpha}_{1}<\widetilde{\alpha}_{2}$;

(2) if $s\left(\widetilde{\alpha}_{1}\right)=s\left(\widetilde{\alpha}_{2}\right)$, then

(i) if $h\left(\widetilde{\alpha}_{1}\right)<h\left(\widetilde{\alpha}_{2}\right)$, then $\widetilde{\alpha}_{1}<\widetilde{\alpha}_{2}$;

(ii) if $h\left(\widetilde{\alpha}_{1}\right)=h\left(\widetilde{\alpha}_{2}\right)$, then $\widetilde{\alpha}_{1} \sim \widetilde{\alpha}_{2}$.

\subsection{Bonferroni Means}

Definition 4 (see [1]). Let $p, q \geq 0$ and $a_{i}(i=1,2, \ldots, n)$ a collection of nonnegative numbers. If

$$
\operatorname{BM}^{p, q}\left(a_{1}, a_{2}, \ldots, a_{n}\right)=\left(\frac{1}{n(n-1)} \sum_{\substack{i, j=1 \\ i \neq j}}^{n} a_{i}^{p} a_{j}^{q}\right)^{1 /(p+q)},
$$

then $\mathrm{BM}^{p, q}$ is called the Bonferroni mean (BM).

Definition 5 (see [6]). Let $p, q, r \geq 0$ and $a_{i}(i=1,2, \ldots, n)$ be a collection of nonnegative numbers. If

$$
\begin{aligned}
\operatorname{GBM}^{p, q, r} & \left(a_{1}, a_{2}, \ldots, a_{n}\right) \\
= & \left(\frac{1}{n(n-1)(n-2)} \sum_{\substack{i, j, k=1 \\
i \neq j \neq k}}^{n} a_{i}^{p} a_{j}^{q} a_{k}^{r}\right)^{1 /(p+q+r)},
\end{aligned}
$$

then $\mathrm{GBM}^{p, q, r}$ is called the generalized Bonferroni mean (GBM).

To deal with intuitionistic fuzzy value and hesitant fuzzy value, $\mathrm{Xu}$ and Yager [12] extended these BMs to fuzzy environment and gave the following concepts.

Definition 6 (see [12]). Let $\alpha_{i}(i=1,2, \ldots, n)$ be a set of intuitionistic fuzzy values. The intuitionistic fuzzy Bonferroni mean (IFBM), the intuitionistic fuzzy weighted Bonferroni mean (IFWBM), and the generalized intuitionistic fuzzy 
weighted Bonferroni mean (GIFWBM) are, respectively, defined as

$$
\begin{aligned}
& \operatorname{IFBM}^{p, q}\left(\alpha_{1}, \alpha_{2}, \ldots, \alpha_{n}\right) \\
& =\left(\frac{1}{n(n-1)} \bigoplus_{\substack{i, j=1, i \neq j}}^{n}\left(\alpha_{i}^{p} \otimes \alpha_{j}^{q}\right)\right)^{1 /(p+q)}, \\
& \operatorname{IFWBM}^{p, q}\left(\alpha_{1}, \alpha_{2}, \ldots, \alpha_{n}\right) \\
& =\left(\frac{1}{n(n-1)} \bigoplus_{\substack{i, j=1, i \neq j}}^{n}\left(\left(w_{i} \alpha_{i}^{p}\right) \otimes\left(w_{j} \alpha_{j}^{q}\right)\right)\right)^{1 /(p+q)}, \\
& \operatorname{GIFBM}^{p, q, r}\left(\alpha_{1}, \alpha_{2}, \ldots, \alpha_{n}\right) \\
& =\left(\frac{1}{n(n-1)(n-2)}\right. \\
& \left.\bigoplus_{\substack{i, j, k=1, i \neq j \neq k}}^{n}\left(\left(w_{i} \alpha_{i}^{p}\right) \otimes\left(w_{j} \alpha_{j}^{q}\right) \otimes\left(w_{k} \alpha_{k}^{r}\right)\right)\right)^{1 /(p+q+r)} .
\end{aligned}
$$

However, it is noted that the above BMs could not effectively aggregate the general fuzzy value, that is, intervalvalued intuitionistic fuzzy value. On the other hand, the above BMs just consider the whole correlationship between the criterion $a_{i}$ and all criteria $\sum_{j=1}^{n} a_{j}^{q} \cdot \sum_{k=1}^{n} a_{k}^{r}$ and cannot reflect the interrelationship between the individual criterion $a_{i}$ and other criteria $v_{i}^{q, r}$ which is the main advantage of the BM. To overcome this drawback, we propose the following OWBM, GOWBM, IIFOWBM, and GIIFOWBM operators.

\section{The Optimized Weighted BM and Its Generalized Form}

The BM and GBM, including IFWBM and GIFWBM, just consider the whole correlationship between the criterion $a_{i}$ and all criteria and cannot reflect the interrelationship between the individual criterion $a_{i}$ and other criteria $v_{i}^{q, r}$ which is the main advantage of the BM. To deal with these issues, in the following subsections, we propose the optimized weighted versions of $\mathrm{BM}$ and its generalized form, that is, the optimized weighted BM (OWBM) and the generalized optimized weighted BM (GOWBM). Based on the Bonferroni mean, we can define the following optimized weighted $\mathrm{BM}(\mathrm{OWBM})$ and generalized optimized weighted BM (GOWBM).

Definition 7. Let $p, q \geq 0$ and $a_{i}(i=1,2, \ldots, n)$ be a collection of nonnegative numbers with the weight vector $w=\left(w_{1}, w_{2}, \ldots, w_{n}\right)$ such that $w_{i} \geq 0$ and $\sum_{i=1}^{n} w_{i}=1$. Then the optimized weighted Bonferroni mean (OWBM) can be defined as follows:

$$
\operatorname{OWBM}^{p, q}\left(a_{1}, a_{2}, \ldots, a_{n}\right)=\left(\sum_{\substack{i, j=1 \\ i \neq j}}^{n} \frac{w_{i} w_{j}}{1-w_{i}} a_{i}^{p} a_{j}^{q}\right)^{1 /(p+q)} .
$$

Definition 8. Let $p, q, r \geq 0$ and $a_{i}(i=1,2, \ldots, n)$ a collection of nonnegative numbers with the weight vector $w=\left(w_{1}, w_{2}, \ldots, w_{n}\right)$ such that $w_{i} \geq 0$ and $\sum_{i=1}^{n} w_{i}=1$. Then the generalized optimized weighted Bonferroni mean (GOWBM) can be defined as follows:

$$
\begin{aligned}
\operatorname{GOWBM}^{p, q, r}\left(a_{1}, a_{2}, \ldots, a_{n}\right) \\
=\left(\sum_{\substack{i, j, k=1 \\
i \neq j \neq k}}^{n} \frac{w_{i} w_{j} w_{k}}{\left(1-w_{i}\right)\left(1-w_{i}-w_{j}\right)} a_{i}^{p} a_{j}^{q} a_{k}^{r}\right)^{1 /(p+q+r)} .
\end{aligned}
$$

Furthermore, we can transform the OWBM and GOWBM into the interrelationship between OWBM and GOWBM forms as follows:

$$
\operatorname{OWBM}^{p, q}\left(a_{1}, a_{2}, \ldots, a_{n}\right)=\left(\sum_{i=1}^{n} w_{i} a_{i}^{p} \sum_{\substack{j=1 \\ j \neq i}}^{n} \frac{w_{j}}{1-w_{i}} a_{j}^{q}\right)^{1 /(p+q)},
$$

$$
\begin{aligned}
& \operatorname{GOWBM}^{p, q, r}\left(a_{1}, a_{2}, \ldots, a_{n}\right) \\
& =\left(\sum_{i=1}^{n} w_{i} a_{i}^{p} \sum_{\substack{j=1 \\
j \neq i}}^{n} \frac{w_{j}}{1-w_{i}} a_{j}^{q} \sum_{\substack{k=1 \\
k \neq i \neq j}}^{n} \frac{w_{k}}{\left(1-w_{i}-w_{j}\right)} a_{k}^{r}\right)^{1 /(p+q+r)} .
\end{aligned}
$$

According to (6)-(7), we see that the terms $\sum_{j=1, j \neq i}^{n}\left(w_{j} /\right.$ $\left.\left(1-w_{i}\right)\right) a_{j}^{q}$ and $\sum_{i, j, k=1, i \neq j \neq k}^{n}\left(w_{i} w_{j} w_{k} /\left(1-w_{i}\right)\left(1-w_{i}-\right.\right.$ $\left.w_{j}\right) a_{j}^{q} a_{k}^{r}$ are the weighted power average satisfaction of all criteria except $A_{i}$, and $\sum_{j=1, j \neq i}^{n}\left(w_{j} /\left(1-w_{i}\right)\right)=1$ and $\sum_{i, j, k=1, i \neq j \neq k}^{n}\left(w_{i} w_{j} w_{k} /\left(1-w_{i}\right)\left(1-w_{i}-w_{j}\right)\right)=1$. If we, respectively, denote the above terms as $u_{i}^{q}$ and $u_{i}^{q} v_{i}^{r}$, thus

$$
\begin{gathered}
\operatorname{OWBM}^{p, q}\left(a_{1}, a_{2}, \ldots, a_{n}\right)=\left(\sum_{i=1}^{n} w_{i} a_{i}^{p} u_{i}^{q}\right)^{1 /(p+q)}, \\
\operatorname{GOWBM}^{p, q, r}\left(a_{1}, a_{2}, \ldots, a_{n}\right)=\left(\sum_{i=1}^{n} w_{i} a_{i}^{p} u_{i}^{q} v_{i}^{r}\right)^{1 /(p+q+r)} .
\end{gathered}
$$

Here then $u_{i}^{q}$ and $u_{i}^{q} v_{i}^{r}$ are the weighted power average satisfaction to all criteria except $A_{i}$, which represents the interrelationship between the individual criterion $a_{i}$ and other criteria $a_{j}(j \neq i)$. This is similar to the BM. 


\section{Two Interval-Valued Intuitionistic Fuzzy BM Operators Based on OWBM and GOWBM}

To aggregate the fuzzy information, $\mathrm{Xu}$ and Yager [12] proposed the IFBM and IFWBM. However, it is noted that the above BMs could not effectively aggregate the general fuzzy value, that is, interval-valued intuitionistic fuzzy value. Thus, we further propose two interval-valued intuitionistic fuzzy $\mathrm{BM}$ operators to aggregate the interval-valued intuitionistic fuzzy correlated information based on the optimized weighted and generalized optimized weighted BMs, respectively, that is, IIFOWBM and GIIFOWBM.

Definition 9. Let $\widetilde{\alpha}_{i}=\left(\left[a_{i}, b_{i}\right],\left[c_{i}, d_{i}\right]\right)$ be a collection of IIFVs with the weight vector $w=\left(w_{1}, w_{2}, \ldots, w_{n}\right)$ such that $p, q \geq$ $0, w_{i} \geq 0$, and $\sum_{i=1}^{n} w_{i}=1$. If

$$
\begin{aligned}
& \operatorname{IIFOWBM}^{p, q}\left(\widetilde{\alpha}_{1}, \widetilde{\alpha}_{2}, \ldots, \widetilde{\alpha}_{n}\right) \\
& \quad=\left(\bigoplus_{\substack{i, j=1, i \neq j}}^{n} \frac{w_{i} w_{j}}{1-w_{i}}\left(\widetilde{\alpha}_{i}^{p} \otimes \widetilde{\alpha}_{j}^{q}\right)\right)^{1 /(p+q)},
\end{aligned}
$$

then IIFOWBM ${ }^{p, q}$ is called the interval-valued intuitionistic fuzzy optimized weighted Bonferroni mean (IIFOWBM).

On the basis of the operational laws of IIFVs, we have the following.

Theorem 10. Letting $\widetilde{\alpha}_{i}=\left(\left[a_{i}, b_{i}\right],\left[c_{i}, d_{i}\right]\right)(i=1,2, \ldots, n)$ be a collection of IIFVs with the weight vector $\left(w_{1}, w_{2}, \ldots, w_{n}\right)$, such that $p, q \geq 0, w_{i} \geq 0$, and $\sum_{i=1}^{n} w_{i}=1$, then the aggregated value by using the IIFOWBM is also an IIFV, and

$$
\begin{aligned}
& \operatorname{IIFOWBM}^{p, q}\left(\widetilde{\alpha}_{1}, \widetilde{\alpha}_{2}, \ldots, \widetilde{\alpha}_{n}\right) \\
& =\left(\bigoplus_{\substack{i, j=1, i \neq j}}^{n} \frac{w_{i} w_{j}}{1-w_{i}}\left(\widetilde{\alpha}_{i}^{p} \otimes \widetilde{\alpha}_{j}^{q}\right)\right)^{1 /(p+q)} \\
& =\left(\left[\left(1-\prod_{\substack{i, j=1, i \neq j}}^{n}\left(1-a_{i}^{p} a_{j}^{q}\right)^{w_{i} w_{j} /\left(1-w_{i}\right)}\right)^{1 /(p+q)},\right.\right. \\
& \left.\left(1-\prod_{\substack{i, j=1, i \neq j}}^{n}\left(1-b_{i}^{p} b_{j}^{q}\right)^{w_{i} w_{j} /\left(1-w_{i}\right)}\right)^{1 /(p+q)}\right] \\
& {\left[1-\left(1-\prod_{\substack{i, j=1, i \neq j}}^{n}\left(1-\left(1-c_{i}\right)^{p}\right.\right.\right.} \\
& \left.\left.\times\left(1-c_{j}\right)^{q}\right)^{w_{i} w_{j} /\left(1-w_{i}\right)}\right)^{1 /(p+q)}
\end{aligned}
$$

$$
\begin{aligned}
1-\left(1-\prod_{\substack{i, j=1, i \neq j}}^{n}\left(1-\left(1-d_{i}\right)^{p}\right.\right. & \\
& \left.\left.\left.\left.\times\left(1-d_{j}\right)^{q}\right)^{w_{i} w_{j} /\left(1-w_{i}\right)}\right)^{1 /(p+q)}\right]\right) .
\end{aligned}
$$

Proof. By the operational laws for IIFVs, we get

$$
\begin{aligned}
& \widetilde{\alpha}_{i}^{p}=\left(\left[a_{i}^{p}, b_{i}^{p}\right],\left[1-\left(1-c_{i}\right)^{p}, 1-\left(1-d_{i}\right)^{p}\right]\right), \\
& \widetilde{\alpha}_{j}^{q}=\left(\left[a_{j}^{q}, b_{j}^{q}\right],\left[1-\left(1-c_{j}\right)^{q}, 1-\left(1-d_{j}\right)^{q}\right]\right), \\
& \widetilde{\alpha}_{i}^{p} \otimes \widetilde{\alpha}_{j}^{q} \\
& =\left(\left[a_{i}^{p} a_{j}^{q}, b_{i}^{p} b_{j}^{q}\right],\right. \\
& \left.\left[1-\left(1-c_{i}\right)^{p}\left(1-c_{j}\right)^{q}, 1-\left(1-d_{i}\right)^{p}\left(1-d_{j}\right)^{q}\right]\right), \\
& \frac{w_{i} w_{j}}{1-w_{i}} \widetilde{\alpha}_{i}^{p} \otimes \widetilde{\alpha}_{j}^{q} \\
& =\left(\left[1-\left(1-a_{i}^{p} a_{j}^{q}\right)^{w_{i} w_{j} /\left(1-w_{i}\right)}, 1-\left(1-b_{i}^{p} b_{j}^{q}\right)^{w_{i} w_{j} /\left(1-w_{i}\right)}\right],\right. \\
& {\left[\left(1-\left(1-c_{i}\right)^{p}\left(1-c_{j}\right)^{q}\right)^{w_{i} w_{j} /\left(1-w_{i}\right)},\right.} \\
& \left.\left.\left(1-\left(1-d_{i}\right)^{p}\left(1-d_{j}\right)^{q}\right)^{w_{i} w_{j} /\left(1-w_{i}\right)}\right]\right), \\
& \bigoplus_{\substack{i, j=1, i \neq j}}^{n} \frac{w_{i} w_{j}}{1-w_{i}} \widetilde{\alpha}_{i}^{p} \otimes \tilde{\alpha}_{j}^{q} \\
& =\left(\left[1-\prod_{\substack{i, j=1, i \neq j}}^{n}\left(1-a_{i}^{p} a_{j}^{q}\right)^{w_{i} w_{j} /\left(1-w_{i}\right)},\right.\right. \\
& \left.1-\prod_{\substack{i, j=1, i \neq j}}^{n}\left(1-b_{i}^{p} b_{j}^{q}\right)^{w_{i} w_{j} /\left(1-w_{i}\right)}\right], \\
& {\left[\prod_{\substack{i, j=1, i \neq j}}^{n}\left(1-\left(1-c_{i}\right)^{p}\left(1-c_{j}\right)^{q}\right)^{w_{i} w_{j} /\left(1-w_{i}\right)},\right.} \\
& \left.\left.\prod_{\substack{i, j=1, i \neq j}}^{n}\left(1-\left(1-d_{i}\right)^{p}\left(1-d_{j}\right)^{q}\right)^{w_{i} w_{j} /\left(1-w_{i}\right)}\right]\right) \text {. }
\end{aligned}
$$


Therefore,

$$
\begin{aligned}
& \operatorname{IIFOWBM}^{p, q}\left(\widetilde{\alpha}_{1}, \widetilde{\alpha}_{2}, \ldots, \widetilde{\alpha}_{n}\right) \\
& \begin{array}{l}
=\left(\bigoplus_{\substack{i, j=1, i \neq j}}^{n} \frac{w_{i} w_{j}}{1-w_{i}}\left(\widetilde{\alpha}_{i}^{p} \otimes \widetilde{\alpha}_{j}^{q}\right)\right)^{1 /(p+q)} \\
=\left(\left[\left(1-\prod_{\substack{i, j=1, i \neq j}}^{n}\left(1-a_{i}^{p} a_{j}^{q}\right)^{w_{i} w_{j} /\left(1-w_{i}\right)}\right)^{1 /(p+q)},\right.\right.
\end{array} \\
& \left.\left(1-\prod_{\substack{i, j=1, i \neq j}}^{n}\left(1-b_{i}^{p} b_{j}^{q}\right)^{w_{i} w_{j} /\left(1-w_{i}\right)}\right)^{1 /(p+q)}\right] \\
& {\left[1-\left(1-\prod_{\substack{i, j=1, i \neq j}}^{n}\left(1-\left(1-c_{i}\right)^{p}\left(1-c_{j}\right)^{q}\right)^{w_{i} w_{j} /\left(1-w_{i}\right)}\right)^{1 /(p+q)},\right.} \\
& 1-\left(1-\prod_{\substack{i, j=1, i \neq j}}^{n}\left(1-\left(1-d_{i}\right)^{p}\right.\right.
\end{aligned}
$$$$
\left.\left.\left.\left.\times\left(1-d_{j}\right)^{q}\right)^{w_{i} w_{j} /\left(1-w_{i}\right)}\right)^{1 /(p+q)}\right]\right)
$$$$
0 \leq\left(1-\prod_{\substack{i, j=1, i \neq j}}^{n}\left(1-a_{i}^{p} a_{j}^{q}\right)^{w_{i} w_{j} /\left(1-w_{i}\right)}\right)^{1 /(p+q)}
$$$$
\leq 1
$$$$
0 \leq\left(1-\prod_{\substack{i, j=1, i \neq j}}^{n}\left(1-b_{i}^{p} b_{j}^{q}\right)^{w_{i} w_{j} /\left(1-w_{i}\right)}\right)^{1 /(p+q)} \leq 1 .
$$

We have

$$
0 \leq 1-\left(1-\prod_{\substack{i, j=1, i \neq j}}^{n}\left(1-\left(1-c_{i}\right)^{p}\left(1-c_{j}\right)^{q}\right)^{w_{i} w_{j} /\left(1-w_{i}\right)}\right)^{1 /(p+q)}
$$

$\leq 1$,
$0 \leq 1-\left(1-\prod_{\substack{i, j=1, i \neq j}}^{n}\left(1-\left(1-d_{i}\right)^{p}\left(1-d_{j}\right)^{q}\right)^{w_{i} w_{j} /\left(1-w_{i}\right)}\right)^{1 /(p+q)}$

$\leq 1$

$$
\begin{aligned}
& \left(1-\prod_{\substack{i, j=1, i \neq j}}^{n}\left(1-b_{i}^{p} b_{j}^{q}\right)^{w_{i} w_{j} /\left(1-w_{i}\right)}\right)^{1 /(p+q)}+1 \\
& -\left(1-\prod_{\substack{i, j=1, i \neq j}}^{n}\left(1-\left(1-d_{i}\right)^{p}\left(1-d_{j}\right)^{q}\right)^{w_{i} w_{j} /\left(1-w_{i}\right)}\right)^{1 /(p+q)}
\end{aligned}
$$

$\leq 1$

$$
\begin{aligned}
& +\left(1-\prod_{\substack{i, j=1, i \neq j}}^{n}\left(1-\left(1-d_{i}\right)^{p}\left(1-d_{j}\right)^{q}\right)^{w_{i} w_{j} /\left(1-w_{i}\right)}\right)^{1 /(p+q)} \\
& -\left(1-\prod_{\substack{i, j=1, i \neq j}}^{n}\left(1-\left(1-d_{i}\right)^{p}\left(1-d_{j}\right)^{q}\right)^{w_{i} w_{j} /\left(1-w_{i}\right)}\right)^{1 /(p+q)} \\
& =1
\end{aligned}
$$

which completes the proof.

Property 1 (idempotency). If all IIFVs $\tilde{\alpha}_{i}=\left(\left[a_{i}, b_{i}\right],\left[c_{i}, d_{i}\right]\right)$ $(i=1,2, \ldots, n)$ are equal, that is, $\widetilde{\alpha}_{i}=\left(\left[a_{i}, b_{i}\right],\left[c_{i}, d_{i}\right]\right)=\widetilde{\alpha}=$ $([a, b],[c, d])$, for all $i$, we have

$$
\operatorname{IIFOWBM}^{p, q}\left(\widetilde{\alpha}_{1}, \widetilde{\alpha}_{2}, \ldots, \widetilde{\alpha}_{n}\right)=\widetilde{\alpha}=([a, b],[c, d]) .
$$

Proof. Since $\widetilde{\alpha}_{i}=\left(\left[a_{i}, b_{i}\right],\left[c_{i}, d_{i}\right]\right)=\widetilde{\alpha}=([a, b],[c, d])$, then

$$
\begin{aligned}
& \operatorname{IIFOWBM}^{p, q}\left(\widetilde{\alpha}_{1}, \widetilde{\alpha}_{2}, \ldots, \widetilde{\alpha}_{n}\right) \\
& =\left(\bigoplus_{\substack{i, j=1, i \neq j}}^{n} \frac{w_{i} w_{j}}{1-w_{i}}\left(\widetilde{\alpha}^{p} \otimes \widetilde{\alpha}^{q}\right)\right)^{1 /(p+q)} \\
& =\left(\widetilde{\alpha}^{p} \otimes \widetilde{\alpha}^{q}\right)^{1 /(p+q)}=\widetilde{\alpha}_{i}
\end{aligned}
$$

which completes the proof of Property 1.

Corollary 11. If $\widetilde{\alpha}_{i}=\left(\left[a_{i}, b_{i}\right],\left[c_{i}, d_{i}\right]\right)(i=1,2, \ldots, n)$ is a collection of the largest IIFVs, that is, $\widetilde{\alpha}_{i}=\tilde{\alpha}=([1,1],[0,0])$, 
for all $i$, and $\left(w_{\sigma(1)}, w_{\sigma(2)}, \ldots, w_{\sigma(n)}\right)$ is precise weight vector of $\widetilde{\alpha}_{i}$, then

$$
\begin{aligned}
& \operatorname{IIFOWBM}^{p, q}\left(\widetilde{\alpha}_{1}, \widetilde{\alpha}_{2}, \ldots, \widetilde{\alpha}_{n}\right) \\
& =\left(\bigoplus_{\substack{i, j=1, i \neq j}}^{n} \frac{w_{i} w_{j}}{1-w_{i}}\left(\widetilde{\alpha}_{i}^{p} \otimes \widetilde{\alpha}_{j}^{q}\right)\right)^{1 /(p+q)} \\
& =\left(\left[\left(1-\prod_{\substack{i, j=1, i \neq j}}^{n}(1-1)\right)^{1 /(p+q)}\right.\right. \\
& \left.\left(1-\prod_{\substack{i, j=1, i \neq j}}^{n}(1-1)\right)^{1 /(p+q)}\right] \\
& \left.\left[1-(1)^{1 /(p+q)}, 1-(1)^{1 /(p+q)}\right]\right) \\
& =([1,1],[0,0])
\end{aligned}
$$

which is also the largest IIFV.

Property 2 (monotonicity). Let $\widetilde{\alpha}_{i}=\left(\left[a_{i}, b_{i}\right],\left[c_{i}, d_{i}\right]\right)$ and $\widetilde{\beta}_{j}=\left(\left[a_{j}, b_{j}\right],\left[c_{j}, d_{j}\right]\right)(i, j=1,2, \ldots, n)$ be two collections of IIFVs; if $a_{i} \leq a_{j}, b_{i} \leq b_{j}, c_{i} \leq c_{j}$ and $d_{i} \leq d_{j}$, for all $i$, then

$$
\begin{aligned}
& \operatorname{IIFOWBM}^{p, q}\left(\widetilde{\alpha}_{1}, \widetilde{\alpha}_{2}, \ldots, \widetilde{\alpha}_{n}\right) \\
& \quad \leq \operatorname{IIFOWBM}^{p, q}\left(\widetilde{\beta}_{1}, \widetilde{\beta}_{2}, \ldots, \widetilde{\beta}_{n}\right) .
\end{aligned}
$$

Proof. The proof of Property 2 is similar to Property 10 in [15].

Property 3 (transformation). Letting $\tilde{\alpha}_{i}=\left(\left[a_{i}, b_{i}\right],\left[c_{i}, d_{i}\right]\right)$ $(i=1,2, \ldots, n)$ be a set of IIFVs and $\left(\bar{\alpha}_{1}, \bar{\alpha}_{2}, \ldots, \bar{\alpha}_{n}\right)$ the permutation of $\left(\widetilde{\alpha}_{1}, \widetilde{\alpha}_{2}, \ldots, \widetilde{\alpha}_{n}\right)$, then

$$
\begin{aligned}
& \operatorname{IIFOWBM}^{p, q}\left(\bar{\alpha}_{1}, \bar{\alpha}_{2}, \ldots, \bar{\alpha}_{n}\right) \\
& \quad=\operatorname{IIFOWBM}^{p, q}\left(\widetilde{\alpha}_{1}, \widetilde{\alpha}_{2}, \ldots, \widetilde{\alpha}_{n}\right) .
\end{aligned}
$$

Proof. Since $\left(\bar{\alpha}_{1}, \bar{\alpha}_{2}, \ldots, \bar{\alpha}_{n}\right)$ is a permutation of $\left(\widetilde{\alpha}_{1}, \widetilde{\alpha}_{2}\right.$, $\left.\ldots, \widetilde{\alpha}_{n}\right)$, then

$$
\begin{gathered}
\left(\bigoplus_{\substack{i, j=1, i \neq j}}^{n} \frac{w_{i} w_{j}}{1-w_{i}} \widetilde{\alpha}_{i}^{p} \otimes \widetilde{\alpha}_{j}^{q}\right)^{1 /(p+q)} \\
=\left(\bigoplus_{\substack{i, j=1, i \neq j}}^{n} \frac{w_{i} w_{j}}{1-w_{i}} \bar{\alpha}_{i}^{p} \otimes \bar{\alpha}_{j}^{q}\right)^{1 /(p+q)} .
\end{gathered}
$$

Thus,

$$
\begin{aligned}
& \operatorname{IIFOWBM}^{p, q}\left(\bar{\alpha}_{1}, \bar{\alpha}_{2}, \ldots, \bar{\alpha}_{n}\right) \\
& =\operatorname{IIFOWBM}^{p, q}\left(\widetilde{\alpha}_{1}, \widetilde{\alpha}_{2}, \ldots, \widetilde{\alpha}_{n}\right) .
\end{aligned}
$$

Definition 12. Let $\tilde{\alpha}_{i}=\left(\left[a_{i}, b_{i}\right],\left[c_{i}, d_{i}\right]\right)(i=1,2, \ldots, n)$ be a collection of IIFVs with the weight vector $w=$ $\left(w_{1}, w_{2}, \ldots, w_{n}\right)$ such that $p, q, r \geq 0$ and $\sum_{i=1}^{n} w_{i}=1$. If

$$
\operatorname{GIIFOWBM}^{p, q, r}\left(\widetilde{\alpha}_{1}, \ldots, \widetilde{\alpha}_{n}\right)
$$

$$
=\left(\bigoplus_{\substack{i, j, k=1 \\ i \neq j \neq k}}^{n} \frac{w_{i} w_{j} w_{k}}{\left(1-w_{i}\right)\left(1-w_{i}-w_{j}\right)}\left(\widetilde{\alpha}_{i}^{p} \otimes \widetilde{\alpha}_{j}^{q} \otimes \widetilde{\alpha}_{k}^{r}\right)\right)^{1 /(p+q+r)},
$$

then GIIFOWBM ${ }^{p, q, r}$ is called the generalized intervalvalued intuitionistic fuzzy optimized weighted Bonferroni mean (GIIFOWBM).

Theorem 13. Letting $\widetilde{\alpha}_{i}=\left(\left[a_{i}, b_{i}\right],\left[c_{i}, d_{i}\right]\right)(i=1,2, \ldots, n)$ be a set of IIFVs with the weight vector $\left(w_{1}, w_{2}, \ldots, w_{n}\right)$, such that $p, q, r \geq 0$ and $\sum_{i=1}^{n} w_{i}=1$, then the aggregated value by using the GIIFOWBM is also an IIFV and

$$
\begin{aligned}
& \operatorname{GIIFOWBM}^{\mathrm{p} q, r}\left(\widetilde{\alpha}_{1}, \widetilde{\alpha}_{2}, \ldots, \widetilde{\alpha}_{n}\right) \\
& =\left(\bigoplus_{\substack{i, j, k=1 \\
i \neq j \neq k}}^{n} \frac{w_{i} w_{j} w_{k}}{\left(1-w_{i}\right)\left(1-w_{i}-w_{j}\right)}\left(\widetilde{\alpha}_{i}^{p} \otimes \widetilde{\alpha}_{j}^{q} \otimes \widetilde{\alpha}_{k}^{r}\right)\right)^{1 /(p+q+r)} \\
& =\left(\left[\left(1-\prod_{\substack{i, j, k=1 \\
i \neq j \neq k}}^{n}\left(1-a_{i}^{p} a_{j}^{q} a_{k}^{r}\right)^{w_{i} w_{j} w_{k} /\left(1-w_{i}\right)\left(1-w_{i}-w_{j}\right)}\right)^{1 /(p+q+r)},\right.\right. \\
& \left.\left(1-\prod_{\substack{i, j, k=1 \\
i \neq j \neq k}}^{n}\left(1-b_{i}^{p} b_{j}^{q} b_{k}^{r}\right)^{w_{i} w_{j} w_{k} /\left(1-w_{i}\right)\left(1-w_{i}-w_{j}\right)}\right)^{1 /(p+q+r)}\right] \\
& \times\left[1-\left(1-\prod_{\substack{i, j, k=1 \\
i \neq j \neq k}}^{n}\left(1-\left(1-c_{i}\right)^{p}\left(1-c_{j}\right)^{q}\right.\right.\right.
\end{aligned}
$$$$
\left.\left.\times\left(1-c_{k}\right)^{r}\right)^{w_{i} w_{j} w_{k} /\left(1-w_{i}\right)\left(1-w_{i}-w_{j}\right)}\right)^{1 /(p+q+r)},
$$$$
1-\left(1-\prod_{\substack{i, j, k=1 \\ i \neq j \neq k}}^{n}\left(1-\left(1-d_{i}\right)^{p}\left(1-d_{j}\right)^{q}\right.\right.
$$$$
\left.\left.\left.\left.\times\left(1-d_{k}\right)^{r}\right)^{w_{i} w_{j} w_{k} /\left(1-w_{i}\right)\left(1-w_{i}-w_{j}\right)}\right)^{1 /(p+q+r)}\right]\right) .
$$ 
Proof. By the operational laws for IIFVs, we have

$$
\begin{aligned}
& \widetilde{\alpha}_{i}^{p}=\left(\left[a_{i}^{p}, b_{i}^{p}\right],\left[1-\left(1-c_{i}\right)^{p}, 1-\left(1-d_{i}\right)^{p}\right]\right), \\
& \widetilde{\alpha}_{j}^{q}=\left(\left[a_{j}^{q}, b_{j}^{q}\right],\left[1-\left(1-c_{j}\right)^{q}, 1-\left(1-d_{j}\right)^{q}\right]\right), \\
& \widetilde{\alpha}_{k}^{r}=\left(\left[a_{k}^{r}, b_{k}^{r}\right],\left[1-\left(1-c_{k}\right)^{r}, 1-\left(1-d_{k}\right)^{r}\right]\right), \\
& \frac{w_{i} w_{j} w_{k}}{\left(1-w_{i}\right)\left(1-w_{i}-w_{j}\right)} \widetilde{\alpha}_{i}^{p} \otimes \widetilde{\alpha}_{j}^{q} \otimes \widetilde{\alpha}_{k}^{r} \\
&=\left(\left[1-\left(1-a_{i}^{p} a_{j}^{q} a_{k}^{r}\right)^{w_{i} w_{j} w_{k} /\left(1-w_{i}\right)\left(1-w_{i}-w_{j}\right)},\right.\right. \\
&\left.1-\left(1-b_{i}^{p} b_{j}^{q} b_{k}^{r}\right)^{w_{i} w_{j} w_{k} /\left(1-w_{i}\right)\left(1-w_{i}-w_{j}\right)}\right], \\
& {\left[\left(1-\left(1-c_{i}\right)^{p}\left(1-c_{j}\right)^{q}\left(1-c_{k}\right)^{r}\right)^{w_{i} w_{j} w_{k} /\left(1-w_{i}\right)\left(1-w_{i}-w_{j}\right)},\right.} \\
&\left(1-\left(1-d_{i}\right)^{p}\left(1-d_{j}\right)^{q}\right. \\
&\left.\left.\left.\quad \times\left(1-d_{k}\right)^{r}\right)^{w_{i} w_{j} w_{k} /\left(1-w_{i}\right)\left(1-w_{i}-w_{j}\right)}\right]\right) .
\end{aligned}
$$

Therefore,

$$
\begin{aligned}
& \operatorname{GIIFOWBM}^{p, q, r}\left(\widetilde{\alpha}_{1}, \widetilde{\alpha}_{2}, \ldots, \widetilde{\alpha}_{n}\right) \\
& =\left(\bigoplus_{\substack{i, j k=1 \\
i \neq j \neq k}}^{n} \frac{w_{i} w_{j} w_{k}}{\left(1-w_{i}\right)\left(1-w_{i}-w_{j}\right)}\left(\widetilde{\alpha}_{i}^{p} \otimes \widetilde{\alpha}_{j}^{q} \otimes \widetilde{\alpha}_{k}^{r}\right)\right)^{1 /(p+q+r)} \\
& =\left(\left[\left(1-\prod_{\substack{i, k=1 \\
i \neq j \neq k}}^{n}\left(1-a_{i}^{p} a_{j}^{q} a_{k}^{r}\right)^{w_{i} w_{j} w_{k} /\left(1-w_{i}\right)\left(1-w_{i}-w_{j}\right)}\right)^{1 /(p+q+r)},\right.\right. \\
& \left.\left(1-\prod_{\substack{i, j k=1 \\
i \neq j \neq k}}^{n}\left(1-b_{i}^{p} b_{j}^{q} b_{k}^{r}\right)^{w_{i} w_{j} w_{k} /\left(1-w_{i}\right)\left(1-w_{i}-w_{j}\right)}\right)^{1 /(p+q+r)}\right], \\
& {\left[1-\left(1-\prod_{\substack{i, j, k=1 \\
i \neq j \neq k}}^{n}\left(1-\left(1-c_{i}\right)^{p}\left(1-c_{j}\right)^{q}\right.\right.\right.} \\
& \left.\left.\times\left(1-c_{k}\right)^{r}\right)^{w_{i} w_{j} w_{k} /\left(1-w_{i}\right)\left(1-w_{i}-w_{j}\right)}\right)^{1 /(p+q+r)}, \\
& 1-\left(1-\prod_{\substack{i, j, k=1 \\
i \neq j \neq k}}^{n}\left(1-\left(1-d_{i}\right)^{p}\left(1-d_{j}\right)^{q}\right.\right. \\
& \left.\left.\left.\left.\times\left(1-d_{k}\right)^{r}\right)^{w_{i} w_{j} w_{k} /\left(1-w_{i}\right)\left(1-w_{i}-w_{j}\right)}\right)^{1 /(p+q+r)}\right]\right) .
\end{aligned}
$$

In addition, since

$$
0 \leq\left(1-\prod_{\substack{i, j, k=1, i \neq j \neq k}}^{n}\left(1-a_{i}^{p} a_{j}^{q} a_{k}^{r}\right)^{w_{i} w_{j} w_{k} /\left(1-w_{i}\right)\left(1-w_{i}-w_{j}\right)}\right)^{1 /(p+q+r)}
$$

$\leq 1$,

$$
0 \leq\left(1-\prod_{\substack{i, j, k=1, i \neq j \neq k}}^{n}\left(1-b_{i}^{p} b_{j}^{q} b_{k}^{r}\right)^{w_{i} w_{j} w_{k} /\left(1-w_{i}\right)\left(1-w_{i}-w_{j}\right)}\right)^{1 /(p+q+r)}
$$

$\leq 1$,

then

$$
\begin{aligned}
0 \leq 1-\left(1-\prod_{\substack{i, j=1, i \neq j}}^{n}\left(1-\left(1-c_{i}\right)^{p}\left(1-c_{j}\right)^{q}\right.\right. \\
\\
\left.\left.\quad \times\left(1-c_{k}\right)^{r}\right)^{w_{i} w_{j} w_{k} /\left(1-w_{i}\right)\left(1-w_{i}-w_{j}\right)}\right)^{1 /(p+q+r)}
\end{aligned}
$$

$\leq 1$,

$$
\begin{aligned}
0 \leq 1-\left(1-\prod_{\substack{i, j=1,1 \\
i \neq j}}^{n}\left(1-\left(1-d_{i}\right)^{p}\left(1-d_{j}\right)^{q}\right.\right. \\
\\
\left.\left.\quad \times\left(1-d_{k}\right)^{r}\right)^{w_{i} w_{j} w_{k} /\left(1-w_{i}\right)\left(1-w_{i}-w_{j}\right)}\right)^{1 /(p+q+r)}
\end{aligned}
$$$$
\leq 1
$$

which completes the proof of Theorem 13.

Property 4 (idempotency). If all IIFVs $\widetilde{\alpha}_{i}=\left(\left[a_{i}, b_{i}\right]\right.$, $\left.\left[c_{i}, d_{i}\right]\right)(i=1,2, \ldots, n)$ are equal, that is, $\widetilde{\alpha}_{i}=$ $\left(\left[a_{i}, b_{i}\right],\left[c_{i}, d_{i}\right]\right)=\widetilde{\alpha}=([a, b],[c, d])$, for all $i$, then

$$
\operatorname{GIIFOWBM}^{p, q, r}\left(\widetilde{\alpha}_{1}, \widetilde{\alpha}_{2}, \ldots, \widetilde{\alpha}_{n}\right)=\widetilde{\alpha}=([a, b],[c, d]) \text {. }
$$

Proof. The proof of Property 4 is similar to Property 1.

Corollary 14. If $\widetilde{\alpha}_{i}=\left(\left[a_{i}, b_{i}\right],\left[c_{i}, d_{i}\right]\right)(i=1,2, \ldots, n)$ is a set of the largest IIFVs, that is, $\widetilde{\alpha}_{i}=\widetilde{\alpha}=([1,1],[0,0])$, for all $i$, and $\left(w_{\sigma(1)}, w_{\sigma(2)}, \ldots, w_{\sigma(n)}\right)$ is precise weight vector of $\widetilde{\alpha}_{i}$, then

$$
\operatorname{GIFOWBM}^{p, q}\left(\widetilde{\alpha}_{1}, \widetilde{\alpha}_{2}, \ldots, \widetilde{\alpha}_{n}\right)=([1,1],[0,0])
$$

which is also the largest IIFV. 
Property 5 (monotonicity). Let $\widetilde{\alpha}_{i}=\left(\left[a_{i}, b_{i}\right],\left[c_{i}, d_{i}\right]\right)$ and $\widetilde{\beta}_{j}=$ $\left(\left[a_{j}, b_{j}\right],\left[c_{j}, d_{j}\right]\right)(i, j=1,2, \ldots, n)$ be two sets of IIFVs; if $a_{i} \leq$ $a_{j}, b_{i} \leq b_{j}, c_{i} \leq c_{j}$ and $d_{i} \leq d_{j}$, for all $i$, then

$$
\begin{aligned}
& \operatorname{GIIFOWBM}^{p, q, r}\left(\widetilde{\alpha}_{1}, \widetilde{\alpha}_{2}, \ldots, \widetilde{\alpha}_{n}\right) \\
& \leq \operatorname{GIIFOWBM}^{p, q, r}\left(\widetilde{\beta}_{1}, \widetilde{\beta}_{2}, \ldots, \widetilde{\beta}_{n}\right) .
\end{aligned}
$$

Proof. The proof of Property 5 is similar to Property 2.

Property 6 (transformation). Letting $\tilde{\alpha}_{i}=\left(\left[a_{i}, b_{i}\right],\left[c_{i}, d_{i}\right]\right)$ $(i=1,2, \ldots, n)$ be a collection of IIFVs and $\left(\bar{\alpha}_{1}, \bar{\alpha}_{2}, \ldots, \bar{\alpha}_{n}\right)$ is any permutation of $\left(\widetilde{\alpha}_{1}, \widetilde{\alpha}_{2}, \ldots, \widetilde{\alpha}_{n}\right)$, then

$$
\begin{aligned}
& \operatorname{GIIFOWBM}^{p, q, r}\left(\bar{\alpha}_{1}, \bar{\alpha}_{2}, \ldots, \bar{\alpha}_{n}\right) \\
& =\operatorname{GIIFOWBM}^{p, q, r}\left(\widetilde{\alpha}_{1}, \widetilde{\alpha}_{2}, \ldots, \widetilde{\alpha}_{n}\right) .
\end{aligned}
$$

Proof. The proof of Property 6 is similar to Property 3.

\section{Case Illustration}

In the following, we will apply the IIFOWBM and GIIFOWBM operators to group decision making and utilize a practical case (adapted from Xu, 2007) [23] involving the assessment of a set of agroecological regions in Hubei Province, China, to illustrate the developed methods.

Located in Central China and the middle reaches of the Changjiang (Yangtze) River, Hubei Province is distributed in a transitional belt where physical conditions and landscapes are on the transition from north to south and from east to west. Thus, Hubei Province is well known as "a land of rice and fish" since the region enjoys some of the favorable physical conditions, with diversity of natural resources and the suitability for growing various crops. At the same time, however, there are also some restrictive factors for developing agriculture such as a tight manland relation between a constant degradation of natural resources and a growing population pressure on land resource reserve. Despite cherishing a burning desire to promote their standard of living, people living in the area are frustrated because they have no ability to enhance the power to accelerate economic development because of a dramatic decline in quantity of natural resources and a deteriorating environment. Based on the distinctness and differences in environment and natural resources, Hubei Province can be roughly divided into four agroecological regions: $a_{1}$-Wuhan-Ezhou-Huanggang; $a_{2}$-Northeast of Hubei; $a_{3}$-Southeast of Hubei; and $a_{4}$-West of Hubei. In order to prioritize these agroecological regions $a_{i}(i=1,2,3,4)$ with respect to their comprehensive functions, a committee comprised of four experts $e_{j}(j=1,2,3,4)$ (whose weight vector is $w=(0.35,0.20,0.15,0.30))$ has been set up to provide assessment information on $a_{i}(i=1,2,3,4)$. The expert $e_{j}$ compared these four agroecological regions with respect to their comprehensive functions and construct and represented the IIFVs $\bar{\alpha}_{i j}=\left(\left[a_{i j}, b_{i j}\right],\left[c_{i j}, d_{i j}\right]\right)$, where $\left[a_{i j}, b_{i j}\right]$ indicates the agreement degree and $\left[c_{i j}, d_{i j}\right]$ indicates the unagreement degree. To get the optimal alternative by the new IIOWBM and GIIFOWBM operators, the following steps are given.
TABLE 1: Interval-valued intuitionistic fuzzy decision matrix $R$.

\begin{tabular}{lcc}
\hline & $a_{1}$ & $a_{2}$ \\
\hline$e_{1}$ & $([0.1595,0.6264]$, & $([0.2034,0.6718]$, \\
& $[0.1707,0.2472])$ & $[0.1539,0.2335])$ \\
$e_{2}$ & $([0.2617,0.6424]$, & $([0.1776,0.7239]$, \\
& $[0.1424,0.2658])$ & $[0.0000,0.1971])$ \\
$e_{3}$ & $([0.2789,0.6039]$, & $([0.3000,0.6120]$, \\
& $[0.1667,0.2643])$ & $[0.1438,0.2877])$ \\
$e_{4}$ & $([0.1971,0.7480]$, & $([0.2827,0.6000]$, \\
& $[0.0000,0.0000])$ & $[0.1668,0.2678])$ \\
\hline \multirow{2}{*}{$e_{1}$} & $a_{3}$ & $([0.3068,0.6319]$, \\
& $([0.2768,0.6693]$, & {$[0.1819,0.2677)$} \\
$e_{2}$ & $[0.1725,0.2672])$ & $([0.2959,0.6343]$, \\
& $([0.2001,0.6744]$, & $[0.1879,0.2493])$ \\
$e_{3}$ & $(0.1175,0.2161])$ & $([0.1941,0.6091]$, \\
& $([0.3492,0.5811]$, & $[0.2127,0.2947])$ \\
$e_{4}$ & $([0.2005,0.3271])$ & $([0.2728,0.5636]$, \\
& $[0.2474,0.2973])$ & $[0.2576,0.3826])$ \\
\hline
\end{tabular}

Step 1. We normalize $\bar{a}_{i j}$ to $r_{i j}$ and construct the normalization interval-valued intuitionistic fuzzy decision matrix $R=$ $\left(r_{i j}\right)_{4 \times 5}($ see Table 1$)$.

Step 2. Aggregate all the preference values $r_{i j}(i, j=1,2,3,4)$ of the $i$ th line and get the overall performance value $c_{i}$ corresponding to the expert $e_{i}$ by the IIFOWBM operator (here we let $p=q=1)$ :

$$
\begin{aligned}
& c_{1}=([0.2326,0.6441],[0.1707,0.2532]), \\
& c_{2}=([0.2472,0.6629],[0.0000,0.2381]), \\
& c_{3}=([0.2702,0.6038],[0.1790,0.2868]), \\
& c_{4}=([0.2467,0.6432],[0.0000,0.0000]) .
\end{aligned}
$$

Step 3. Calculating the score $s_{2}\left(c_{i}\right)$ of $c_{i}(i=1,2,3,4)$, respectively, we can get

$$
\begin{array}{ll}
s_{2}\left(c_{1}\right)=0.6132, & s_{2}\left(c_{2}\right)=0.6680 \\
s_{2}\left(c_{3}\right)=0.6021, & s_{2}\left(c_{4}\right)=0.7225 .
\end{array}
$$

Therefore,

$$
s_{2}\left(c_{4}\right)>s_{2}\left(c_{2}\right)>s_{2}\left(c_{1}\right)>s_{2}\left(c_{3}\right)
$$

and $a_{4}>a_{2}>a_{1}>a_{3}$, and $y_{4}$ is still the optimal alternative.

Based on GOWBM and GIIFOWBM operators, the main steps are as follows.

Step $1^{\circ}$. See Step 1 .

Step $2^{\circ}$. See Step 2 .

Step $3^{\circ}$. Utilize the GOWBM and GIIFOWBM operators to aggregate all the interval-valued individual intuitionistic 
TABLE 2: Interval-valued intuitionistic fuzzy decision matrix of $D$.

\begin{tabular}{lcc}
\hline & $a_{1}$ & $a_{2}$ \\
\hline$e_{1}$ & $([0.1348,0.5504]$, & $([0.1705,0.6424]$, \\
& $[0.2320,0.3113])$ & $[0.1761,0.2825])$ \\
$e_{2}$ & $([0.2279,0.6064]$, & $([0.1555,0.6917]$, \\
& $[0.1524,0.2832])$ & $[0.1006,0.2293])$ \\
$e_{3}$ & $([0.2742,0.5941]$, & $([0.3000,0.6020]$, \\
& $[0.1749,0.2806])$ & $[0.1539,0.3120])$ \\
$e_{4}$ & $([0.1769,0.6868]$, & $([0.2657,0.6000]$, \\
& $[0.1530,0.2183])$ & $[0.2109,0.2846])$ \\
\hline \multirow{2}{*}{$e_{1}$} & $a_{3}$ & $([0.2924,0.6154]$, \\
& $([0.2112,0.6360]$, & $[0.1998,0.2733])$ \\
$e_{2}$ & $(0.2134,0.2834])$ & $([0.2469,0.5951]$, \\
& $([0.1832,0.6459]$, & $[0.2188,0.2995])$ \\
$e_{3}$ & $([0.1243,0.2528])$ & $([0.1770,0.4885]$, \\
& $[0.2259,0.3477])$ & $[0.2534,0.3349])$ \\
$e_{4}$ & $([0.2196,0.5028]$, & $([0.2427,0.5387]$, \\
& $[0.3157,0.3367])$ & $[0.3145,0.3915])$ \\
\hline
\end{tabular}

fuzzy decision matrices $D_{(q)}=\left(d_{q}^{i j}\right)_{4 \times 4}(q=1,2,3,4)$ into the collective interval-valued intuitionistic fuzzy decision matrix $D=\left(d_{i j}\right)_{4 \times 4}($ see Table 2$)$.

Step 4. Aggregate all the preference values $d_{i j}(i, j=1,2,3,4)$ of the $i$ th line and get the overall performance value $c_{i}$ corresponding to the expert $e_{i}$ by the GIIFOWBM operator (here we let $p=q=r=1$ ):

$$
\begin{aligned}
& c_{1}=([0.1907,0.5999],[0.2086,0.2901]), \\
& c_{2}=([0.2093,0.6250],[0.1589,0.2732]), \\
& c_{3}=([0.2532,0.5593],[0.2029,0.3137]), \\
& c_{4}=([0.2179,0.5931],[0.2409,0.3050]) .
\end{aligned}
$$

Step 5. Calculating the score $s_{2}\left(c_{i}\right)$ of $c_{i}(i=1,2,3,4)$, respectively, we have

$$
\begin{array}{ll}
s_{2}\left(c_{1}\right)=0.5729, & s_{2}\left(c_{2}\right)=0.6005, \\
s_{2}\left(c_{3}\right)=0.5740, & s_{2}\left(c_{4}\right)=0.5663 .
\end{array}
$$

Therefore,

$$
s_{2}\left(c_{2}\right)>s_{2}\left(c_{3}\right)>s_{2}\left(c_{1}\right)>s_{2}\left(c_{4}\right)
$$

and $a_{2}>a_{3}>a_{1}>a_{4}$, and $y_{4}$ is still the optimal alternative.

Based on the previous analysis, it could be found that the most comprehensive function is the West of Hubei.

It should be noted out that the whole ranking of the alternatives has changed. The IIFOWBM ${ }^{1,1}$ produces the ranking of all the alternatives as $a_{4}>a_{2}>a_{1}>a_{3}$, which is slightly different from the ranking of alternatives $a_{2}>a_{3}>a_{1}>$ $a_{4}$, derived by the GIIFOWBM ${ }^{1,1,1}$. Therefore, we can see that the value derived by the IIFOWBM or GIIFOWBM operators depends on the choice of the parameters $p, q$, and $r$.

\section{Concluding Remarks}

To further develop the BM, we have proposed the optimized weighted Bonferroni mean (OWBM) and the generalized optimized weighted Bonferroni mean (GOWBM) in this paper. Then, we proposed two new BM operators under the interval-valued intuitionistic fuzzy environment, that is, the interval-valued intuitionistic fuzzy optimized weighted Bonferroni mean (IIFOWBM) and the generalized intervalvalued intuitionistic fuzzy optimized weighted Bonferroni mean (GIIFOWBM). The new BMs can reflect the preference and interrelationship of the aggregated arguments and can satisfy the basic properties of the aggregation techniques simultaneously. Furthermore, some desirable properties of the IFIOWBM and GIIFOWBM operators are investigated in detail, including idempotency, monotonicity, transformation, and boundary. Finally, based on IIFOWBM and GIIFOWBM, we give a utilized practical case involving the assessment of a set of agroecological regions in China to illustrate these new BMs.

\section{Acknowledgment}

This work was supported by National Basic Research Program of China (973 Program, no. 2010 CB328104-02).

\section{References}

[1] C. Bonferroni, "Sulle medie multiple di potenze," vol. 5, pp. 267270, 1950.

[2] R. R. Yager, "On generalized Bonferroni mean operators for multi-criteria aggregation," International Journal of Approximate Reasoning, vol. 50, no. 8, pp. 1279-1286, 2009.

[3] G. Choquet, "Theory of capacities," Annales de l'Institut Fourier, vol. 5, pp. 131-295, 1955.

[4] R. R. Yager, "On ordered weighted averaging aggregation operators in multicriteria decisionmaking," IEEE Transactions on Systems, Man, and Cybernetics, vol. 18, no. 1, pp. 183-190, 1988.

[5] J. Mordelová and T. Rückschlossová, "ABC-aggregation functions," in Proceedings of the 5th International Summer School on Aggregation Operators, pp. 167-170, Palmade Mallorca, Spain, 2009.

[6] G. Beliakov, S. James, J. Mordelová, T. Rückschlossová, and R. R. Yager, "Generalized Bonferroni mean operators in multicriteria aggregation," Fuzzy Sets and Systems, vol. 161, no. 17, pp. 2227-2242, 2010.

[7] L. A. Zadeh, "Fuzzy sets," Information and Computation, vol. 8, pp. 338-353, 1965.

[8] L. A. Zadeh, "The concept of a linguistic variable and its application to approximate reasoning. I," Learning Systems and Intelligent Robots, vol. 8, pp. 199-249, 1975.

[9] K. T. Atanassov, "Intuitionistic fuzzy sets," Fuzzy Sets and Systems, vol. 20, no. 1, pp. 87-96, 1986.

[10] K. Atanassov and G. Gargov, "Interval valued intuitionistic fuzzy sets," Fuzzy Sets and Systems, vol. 31, no. 3, pp. 343-349, 1989.

[11] V. Torra, "Hesitant fuzzy sets," International Journal of Intelligent Systems, vol. 25, no. 6, pp. 529-539, 2010.

[12] Z. Xu and R. R. Yager, "Intuitionistic fuzzy bonferroni means," IEEE Transactions on Systems, Man, and Cybernetics B, vol. 41, no. 2, pp. 568-578, 2011. 
[13] Z. Xu and Q. Chen, "A multi-criteria decision making procedure based on interval-valued intuitionistic fuzzy bonferroni means," Journal of Systems Science and Systems Engineering, vol. 20, no. 2, pp. 217-228, 2011.

[14] M. Xia, Z. Xu, and B. Zhu, "Generalized intuitionistic fuzzy Bonferroni means," International Journal of Intelligent Systems, vol. 27, no. 1, pp. 23-47, 2012.

[15] W. Zhou and J. M. He, "Intuitionistic fuzzy geometric bonferroni means and their application in Multi-Criteria decision making," International Journal of Intelligent Systems, vol. 27, pp. 995-1019, 2012.

[16] G. Beliakov and S. James, "Defining Bonferroni means over lattices," in Proceedings of the IEEE International Conference on Fuzzy Systems, pp. 1-8, 2012.

[17] W. Zhou and J. M. He, "Intuitionistic fuzzy Normalized weighted Bonferroni mean and its application in multiCriteria decision making," Journal of Applied Mathematics, vol. 2012, Article ID 136254, 22 pages, 2012.

[18] M. M. Xia, Z. S. Xu, and B. Zhu, "Geometric Bonferroni means with their application in multi-criteria decision making," Knowledge-Based Systems, vol. 40, pp. 99-100, 2013.

[19] G. Beliakov and S. James, "On extending generalized Bonferroni means to Atanassov orthopairs in decision making contexts," Fuzzy Sets and Systems, vol. 211, pp. 84-98, 2013.

[20] Z. Xu, "Intuitionistic fuzzy aggregation operators," IEEE Transactions on Fuzzy Systems, vol. 15, no. 6, pp. 1179-1187, 2007.

[21] D. G. Park, Y. C. Kwun, J. H. Park, and I. Y. Park, "Correlation coefficient of interval-valued intuitionistic fuzzy sets and its application to multiple attribute group decision making problems," Mathematical and Computer Modelling, vol. 50, no. 9-10, pp. 1279-1293, 2009.

[22] C. Tan, "A multi-criteria interval-valued intuitionistic fuzzy group decision making with Choquet integral-based TOPSIS," Expert Systems with Applications, vol. 38, no. 4, pp. 3023-3033, 2011.

[23] Z. Xu, "Intuitionistic preference relations and their application in group decision making," Information Sciences, vol. 177, no. 11, pp. 2363-2379, 2007. 


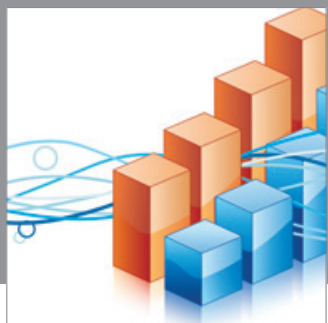

Advances in

Operations Research

mansans

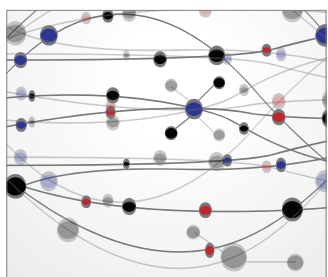

The Scientific World Journal
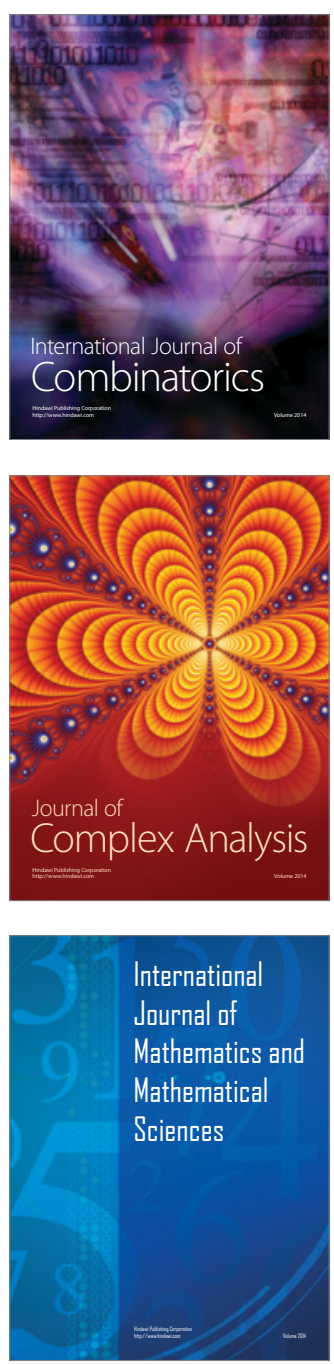
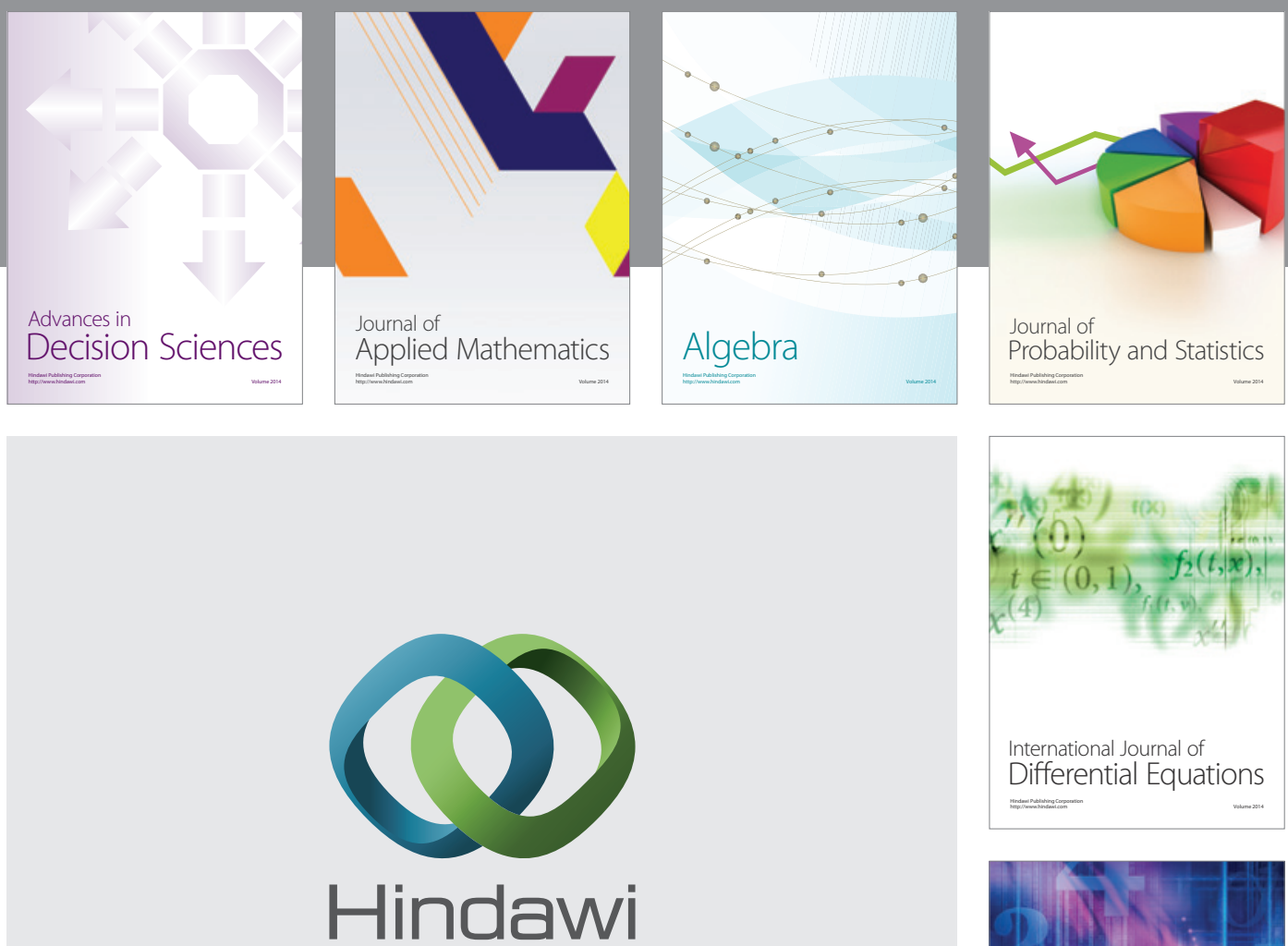

Submit your manuscripts at http://www.hindawi.com
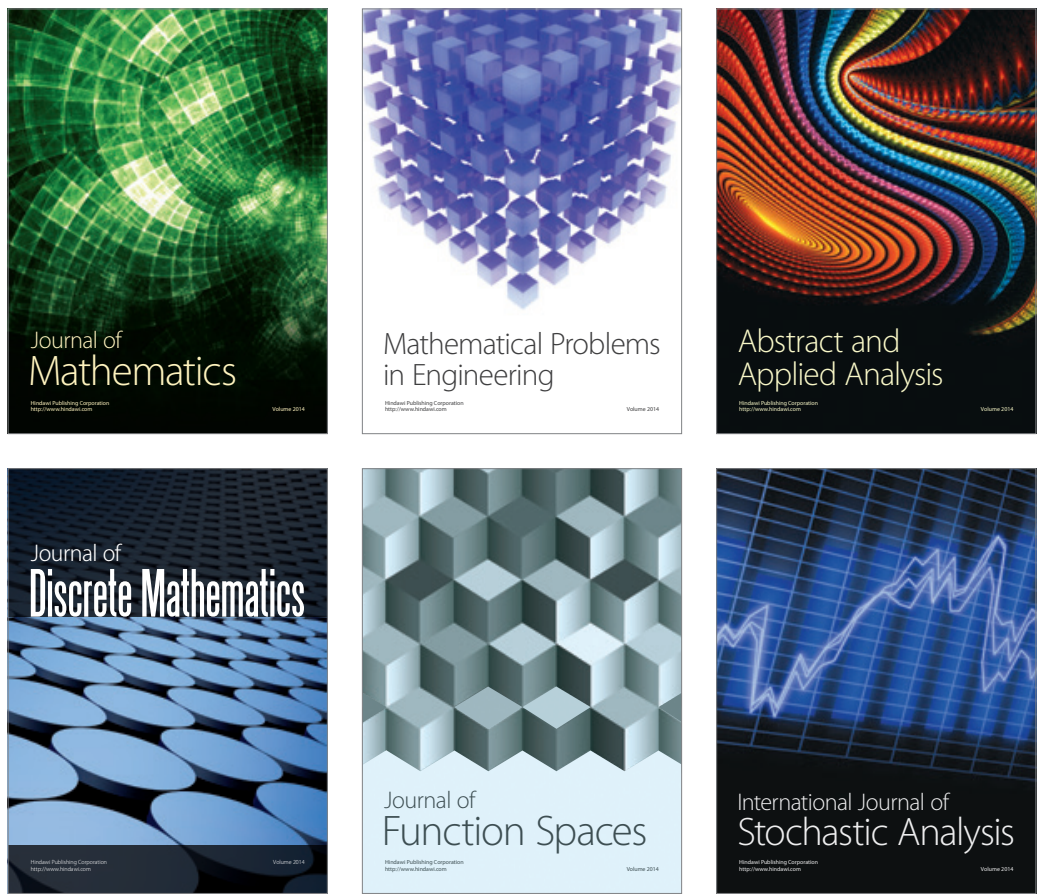

Journal of

Function Spaces

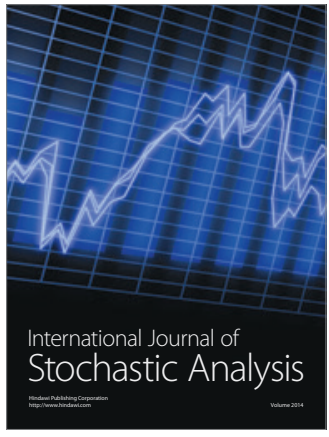

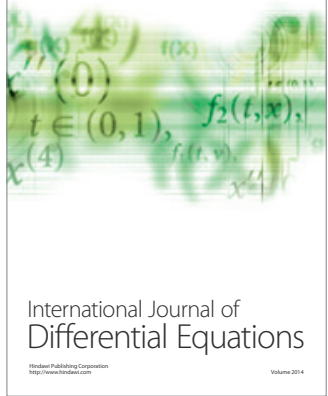
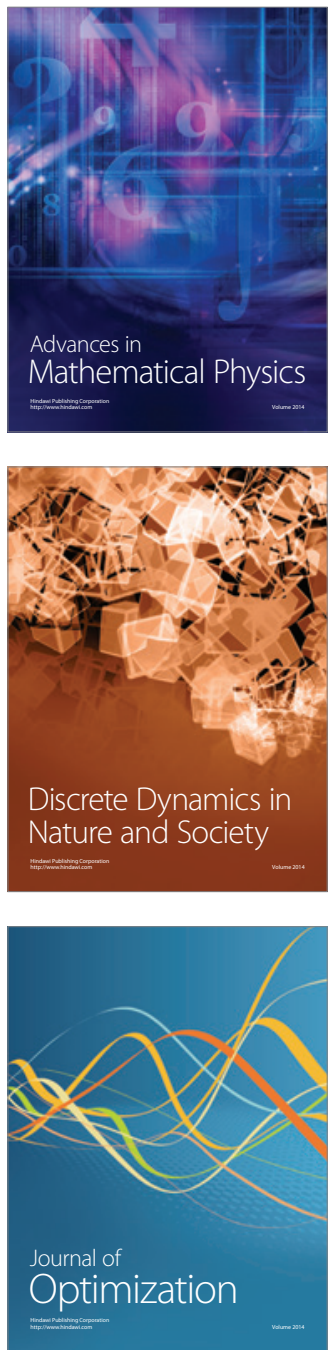